Sitientibus Série Ciências Físicas 09: 1-12 (2013)

\title{
Sistema Automático para Imagens Magnéticas Bidimensionais
}

\author{
An Automatic System for Two-Dimensional Magnetic Images
}

\author{
T.N. Santos \\ Instituto de Física - UFBA \\ Campus Universitário de Ondina \\ Salvador - BA - 40210-340
}

A.R. Barboni
Centro de Referência de Informação em Saúde (CRIS) - Departamento de Saúde - UEFS
Av. Transnordestina, $s / n, k m$ 03, BR 116
Feira de Santana - BA - 44036-900

\author{
E.M. Mascarenhas, M.O. Costa. ${ }^{*}$ E.S. Ferreira, e J.A. Leyva-Cru话 \\ Laboratório de Instrumentação em Física (LINFIS) - Departamento de Física - UEFS \\ Av. Transnordestina, $\mathrm{s} / \mathrm{n}, \mathrm{km}$ 03, $\mathrm{BR} 116$ \\ Feira de Santana - BA - 44036-900
}

\begin{abstract}
Apresenta-se um protótipo de Scanner magnético automatizado para a obtenção de imagens magnéticas bidimensionais $(2 D)$, usando um sensor magnetorresistivo. Estes magnetômetros convertem sinais magnéticos em tensão elétrica diferencial. A automação foi realizada por meio de uma rotina em LabVIEW 8.6, e o processamento digital e a obtenção das imagens magnéticas, realizados off-line, usando uma rotina em linguagem MATLAB 7.6. O número de amostras foi de 400 amostras, adquiridas com uma frequência de aquisição de $4 \mathrm{kHz}$. Foram realizados vários experimentos usando fantomas magnéticos. Os resultados preliminares mostram que o sistema é capaz de obter sinais e imagens magnéticas de diferentes características, varrendo áreas de dimensões até $(0.12 \times 0.12) \mathrm{m}^{2}$.
\end{abstract}

Palavras-chaves: Imagens Magnéticas, Instrumentação, Sensores Magnéticos.

We present a prototype of an automated magnetic scanner for obtaining two-dimensional magnetic image $(2 D)$, using a magnetoresistive sensor. This magnetometer converts magnetic signals in differential voltage. A routine implemented in LabVIEW language performs the automatic control of the experimental system while the digital processing as well as the magnetic images formation, performed off-line, were realized through a routine written in Programming Language MATLAB 7.6. The number of samples was 400 samples and the sample frequency was $4 \mathrm{kHz}$. Several experiments were performed using magnetic phantoms. Preliminary results show that the system is able to obtain signals and images of different magnetic characteristics, with the ability to scan areas up to sizes $(0.12 \times 0.12) \mathrm{m}^{2}$.

Key-words: Magnetic Images, Instrumentation, Magnetic Sensors.

\section{INTRODUÇÃO}

$\mathrm{Na}$ atualidade existem numerosas técnicas de formação de imagens sendo aplicadas em várias áreas da vida do homem, e cada uma

\footnotetext{
*Bolsistas de IC do LINFIS:

${ }^{\dagger}$ Endereço Eletrônico: jalbertoleyva@yahoo.com.br
}

delas funciona com um princípio físico diferente. Dentro destas podemos destacar: o uso dos Raios- $X$ na Tomografia Computadorizada (TC) multi-slice [1, 2]; a detecção de campos magnéticos de amplitudes extremamente baixas, gerados pela atividade síncrona dos neurônios no córtex cerebral na Magnetoencefalografia (MEG) 3-6]; a Tomografia por Imagens de Ressonância Magnética Nuclear (MRI) 
[7, 8], na qual se tira proveito do fenômeno de ressonância entre os átomos de spin semiinteiro e os campos eletromagnéticos na faixa de radiofrequência; a Ultrassonografia e Efeito Doppler Colorido, na qual ondas de som de alta frequência são enviadas ao interior do corpo humano e, após serem refletidas pelas fronteiras entre os tecidos ou pelos líquidos em movimentos, são detectadas e convertidas em imagens [9, 10]; e a Tomografia por Emissão de Pósitrons (PET), uma das técnicas mais robustas da atualidade, a qual faz uso da radiação ionizante de alta energia (Raios- $\gamma$ ) emitida por rádio-fármacos previamente introduzidos no organismo, cumprindo a famosa lei de Einstein $E=m c^{2}$ [11, 12]. Ainda em fases de pesquisas, existem várias técnicas experimentais, como por exemplos, as técnicas de Tomografias de Indução Magnéticas (TIM) [13 15, Método de Tomografia Magnética (MTM) [1621], entre outras 22].

O presente trabalho tem como principal objetivo a construção de um Scanner magnético usando um sensor magnetorresistivo para obtenção de imagens magnéticas em duas dimensões de baixa amplitude e geradas por um dipolo magnético.

\section{MÉTODOS E MATERIAIS}

\section{A. Fantomas Magnéticos}

Os fantomas aqui usados foram basicamente ímãs permanentes de dois tipos. Os de elevado campo magnético chamados de Ímãs de Neodímio, compostos por uma liga de neodímio, ferro e boro $\left(N d_{2} F e_{14} B\right)$. Estes ímãs possuem um alto produto energético comparado a sua massa, alta resistência a desmagnetização e altíssima indução residual, sendo na sua superfície na ordem de 1,2 T. Os outros tipos de ímãs são formados por materiais cerâmicos a base de oxido de ferro. Neste casso usamos ímãs de Ferrite ou Ferrita $\left(\mathrm{Fe}_{3} \mathrm{O}_{4}\right)$. Desde o ponto de vista das pro- priedades magnéticas, estes materiais são classificados como ferrimagnéticos.

As Ferritas podem ser classificadas como materiais magneticamente fracos ou duros. No primeiro caso apresentam baixo valor do campo coercitivo. Isto significa que a direção da magnetização destes materiais pode ser revertida facilmente sem dissipar muita energia (curva de histerese estreita). Ao contrário das ferritas moles, as ferritas duras apresentam um valor elevado do campo coercitivo, o qual significa alto valor do campo remanente depois da magnetização.

Na Fig. 1, são mostradas as fotos e algumas representações esquemáticas dos polos norte e sul dos ímãs utilizados como fantomas nos experimentos realizados neste trabalho. Na Fig. 1.1a, ilustramos um ímã de Neodímio com geometria em forma de anel $(\oslash e 19 \times \oslash i 8 \times h 3)$ mm. Na Fig. 1.1b, observamos um ímã de Ferrite em formato de anel $(\oslash e 44 \times \oslash i 42 \times h 9) \mathrm{mm}$, nas figuras $1.1 c-e$ mostram-se os ímãs cilíndro de Neodímio $(\oslash 2 \times h 4) \mathrm{mm}$, um ímã de Ferrite em bloco $(24 \times 14 \times 5) \mathrm{mm}$ com orifício central de diâmetro de $5 \mathrm{~mm}$, e outro ímã de Ferrite em bloco de duas camadas com dimensões $(40 \times 18 \times 6) \mathrm{mm}$. Vale salientar que os ímãs de Ferrite eram compostos de Ferrite duro.

\section{B. Driver de Potência para os Motores de Passo}

Para acionarmos um motor de passo, precisamos de um hardware específico, chamado driver de potência, cuja função principal é aumentar a tensão recebida para a alimentação requerida pelos motores. Os drivers podem ser construídos utilizando transistores de potência. No entanto, a forma mais fácil é adquirir um driver pronto utilizando um circuito integrado o circuito integrado ULN2003.

Este componente eletrônico é uma matriz de transistores TTL, tipo Darlington, de 7 bits, 50 $\mathrm{V}$, que pode controlar correntes de até $500 \mathrm{~mA}$, sendo de baixo custo e pode ser encontrado em alguns dispositivos como impressoras. Em virtude da circulação de corrente relativamente al- 
tas no driver do motor, foi confeccionado, junto ao circuito de controle, um acoplamento ótico. Para isso, foram utilizados acopladores óticos do tipo QTC $4 N 35$, que garantem o isolamento físico da porta paralela com o driver do motor.

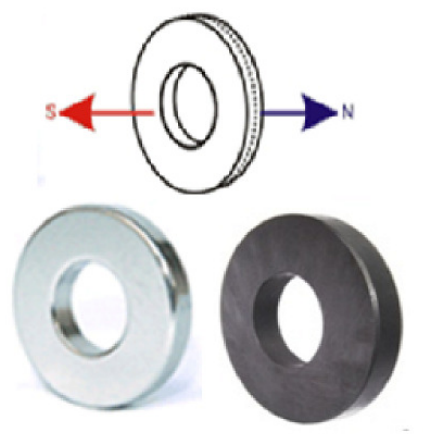

(a)

(b)

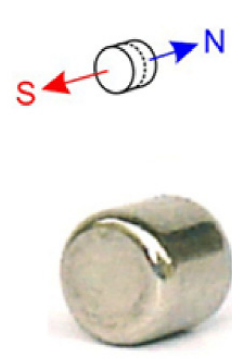

(c)

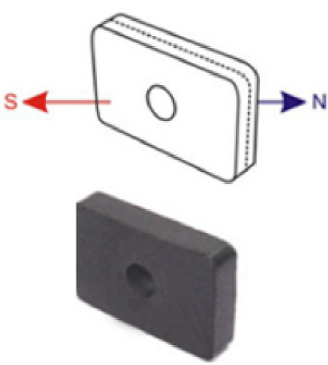

(d)

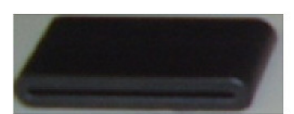

(e)

FIGURA 1: Ímãs permanentes usados como fantomas, em (a) ímã de Neodímio em forma de anel, em (b) um ímã de Ferrite em anel, em (c) ímã cilindro de NdBFe, em (d) um ímã de Ferrite em forma bloco com orifício de $5 \mathrm{~mm}$, e em (e) um bloco de Ferrite de duas camadas.

Na Fig. 2, podemos ver o diagrama esquemático do driver ou circuito de controle dos motores de passo do sistema de posicionamento motorizado do Scanner Magnético. Nesse esquema podemos ver as diferentes ligações entre os diversos componentes eletrônicos usados, destacando-se os CI's e o conector DB-25. Através deste último são enviados pulsos digitais $(0 V / 5 V)$ para o driver. Também pode ser visto o sistema de proteção da porta paralela com os acopladores óticos. A verificação de envio (presença) dos pulsos digitais é feita com o uso dos LED's (4 led's verdes para um motor e 4 led's vermelhos para outro motor) que são acionados cada vez que um sinal digital ou pulso é escrito via software nos pinos ou portos da porta paralela. Logo em seguida, os CIULN2003 potencializam os sinais que, de forma controlada, ativam as 4-fases dos motores (processo de alimentação controlada) e, assim, se dá o controle dos mesmos.

\section{Esquema de Medição do Scanner Magnético}

Na Fig. 3a, mostramos o diagrama esquemático do processo de medição do Scanner Magnético durante a obtenção de imagens magnéticas. Nela observamos a representação gráfica de uma fonte magnética (imã de Neodímio $N d F e B$ ), do detector magnético situado a uma dada distância da fonte magnética, chamada de distância de Liftoff, e as duas direções $(X, Y)$ de varredura.

Na Fig. 3b, observamos uma representação gráfica da metodologia utilizada para fazer as medições do campo magnético em função do espaço em duas dimensões. As medidas são realizadas num set de pontos discretos acima da amostra, este processo é chamado de discretização. Inicialmente, o sistema executa um passo na direção- $X$ (sem medir) e, logo em seguida, faz uma varredura em toda a direção- $Y$, nesta direção para cada passo, o 
sensor realiza a leitura de 400 amostras, com uma taxa de aquisição de $4 \mathrm{kHz}$. No eixo- $X$, foi escolhido um período de amostragem ou re- solução de $0,184 \mathrm{~mm}$, e no eixo- $Y$ foi escolhida um valor de $1,3 \mathrm{~mm}$.

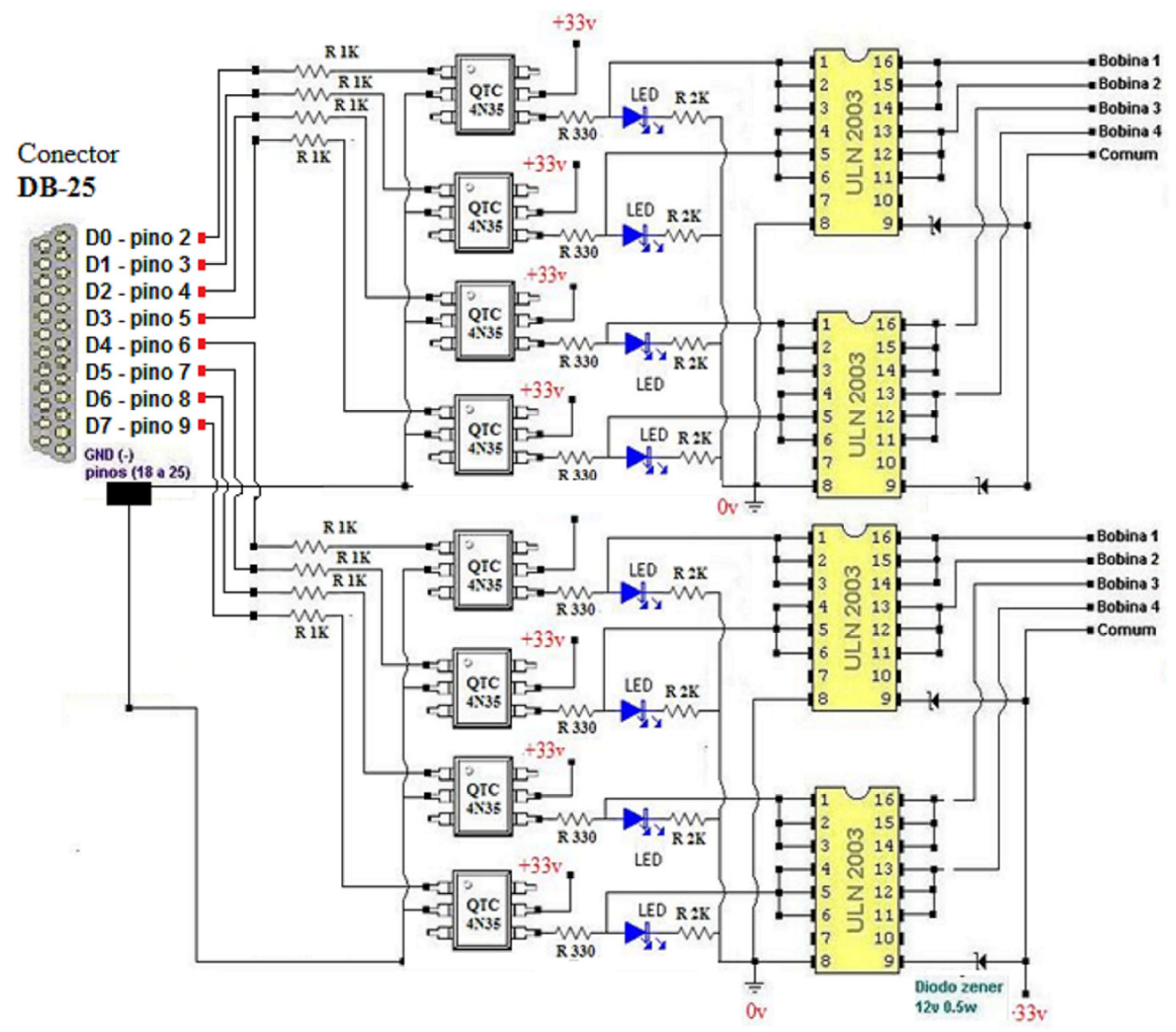

FIGURA 2: Driver do circuito de controle dos dois motores de passo, utilizados no sistema de posicionamento motorizado do Scanner Magnético.

\section{Circuito de Condicionamento de Sinais}

Uma etapa importante na montagem do Scanner Magnético é aquela formada pelo sensor magnético e o circuito de condicionamento do sinal analógico, que é gerado pelo próprio sensor em resposta ao estímulo físico que atua sobre ele (nosso casso é o campo magnético), o qual é de nosso interesse medir. Um sensor magnético também pode ser considerado um transdutor de energia magnética em energia elétrica. Nesta pesquisa, o sensor utilizado é o magnetômetro magnetorresistivo, modelo KMZ10A da Philips. Por causa dos ruídos de natureza eletromagnética presentes no Laboratório e, das variações da tensão elétrica gera- 
das na saída do sensor magnético serem da ordem de alguns milivolts, é necessária a criação de uma etapa de filtragem seletiva e de ampli- ficação do sinal, principalmente quando se faz uso de um amplificador de instrumentação.

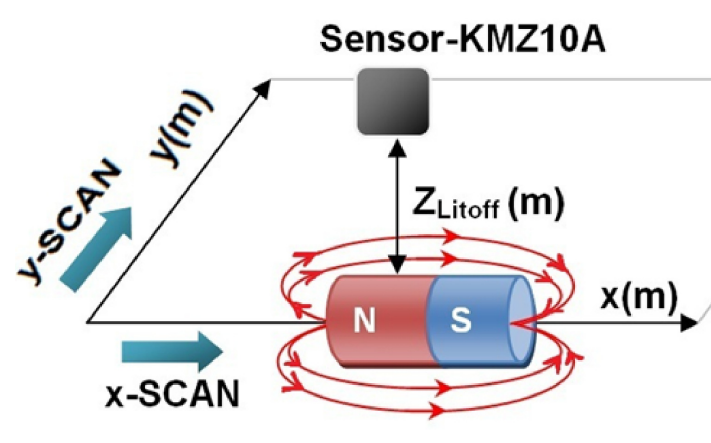

(a)

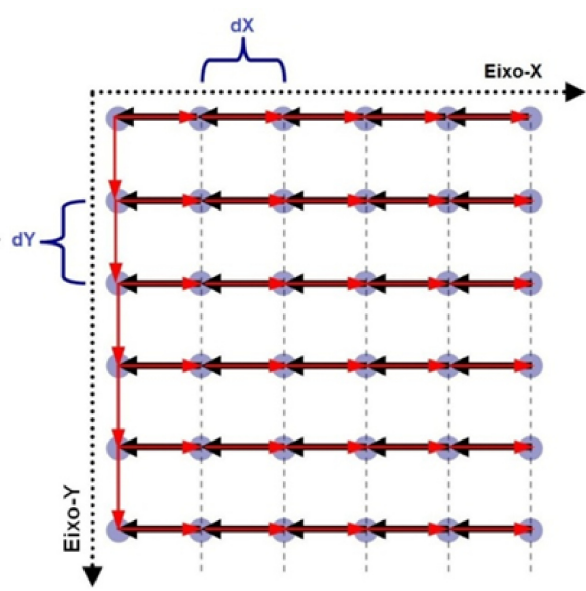

(b)

FIGURA 3: (a) Diagrama esquemático do Scanner Magnético durante o processo de obtenção de imagens magnéticas e para o estudo da dependência do campo magnético com a posição, (b) Discretização da região a ser imageada.

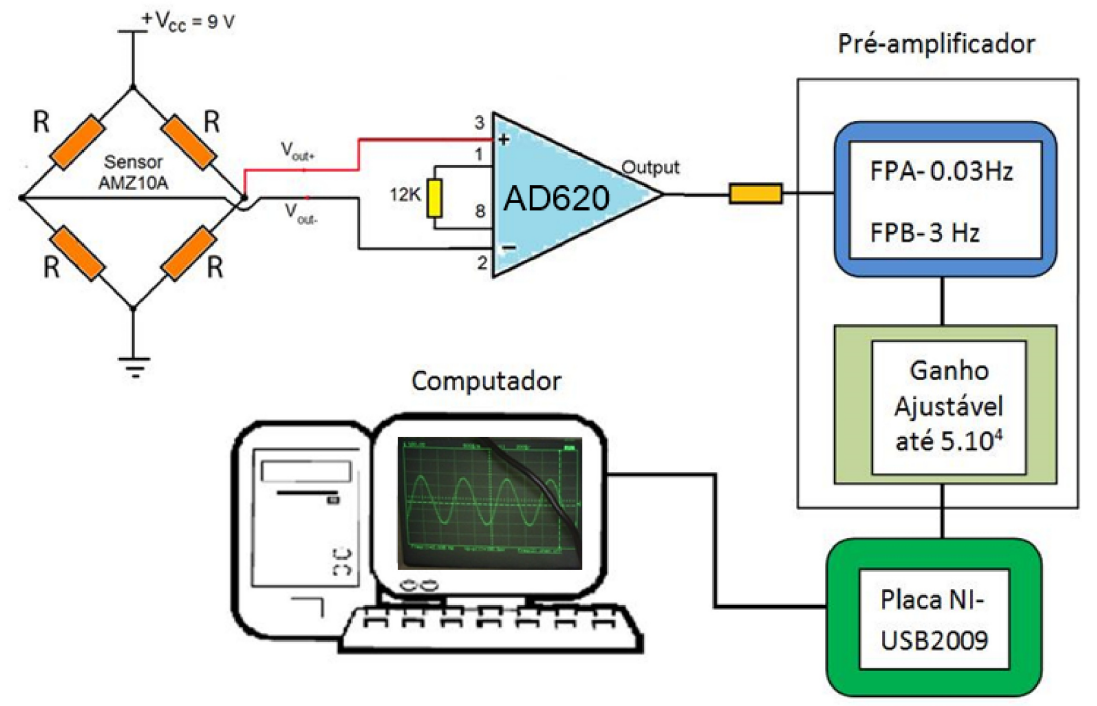

FIGURA 4: Diagrama em bloco do circuito de condicionamento do sinal proveniente do sensor magnético utilizado no sistema experimental. O principal componente é o amplificador de instrumentação AD620. 
Na Fig. 4, mostramos o diagrama em bloco do circuito de condicionamento dos sinais, baseado no amplificador de instrumentação AD620 da Analogical Devices. Este circuito integrado foi escolhido porque apresenta, como uma de suas principais características, uma taxa de rejeição em modo comum mínimo de $100 \mathrm{~dB}$, além de ter uma densidade de ruído de entrada de $9 \mathrm{nV} /(\mathrm{Hz})-1 / 2$ e, na banda de baixas frequências $(0.1 \mathrm{~Hz}$ até $10 \mathrm{~Hz})$, que é uma das faixas de frequências de nosso interesse, o ruído é da ordem de $0.28 \mu \mathrm{V}$ pico a pico. Desta forma, o sensor poderia captar variações na ordem de nanotesla, com ultrabaixo ruído. Os cabos usados para alimentar e conduzir os sinais de saída do sensor foram coaxiais com blindagem ao ruído eletromagnético.

\section{E. Scanner Magnético}

Na Fig. 5, mostra-se uma foto do sistema experimental desenvolvido, composto por o sistema mecânico de posicionamento- $X Y$ (executado por dois motores de passo) (9), um driver de alimentação dos motores (7), um sensor magnetorresistivo (modelo KMZ10A) (10), um par de baterias de $12 \mathrm{~V}$ (8), uma etapa de condicionamento analógico (6), que inclui um amplificador de instrumentação (AD620AN) com ganho fixo de $4 \times$ e um préamplificador (modelo SR560, da Stanford Research System) (3), de elevado desempenho e de ultrabaixo ruído, com ganho ajustável. A filtragem analógica foi personalizada para eliminar ruídos de baixa e alta frequência, escolhendo-se as frequências de corte, ou cutoff, com valores de $0.03 \mathrm{~Hz}$ para o filtro passa alta e $1 \mathrm{~Hz}$ para o filtro passa baixa.

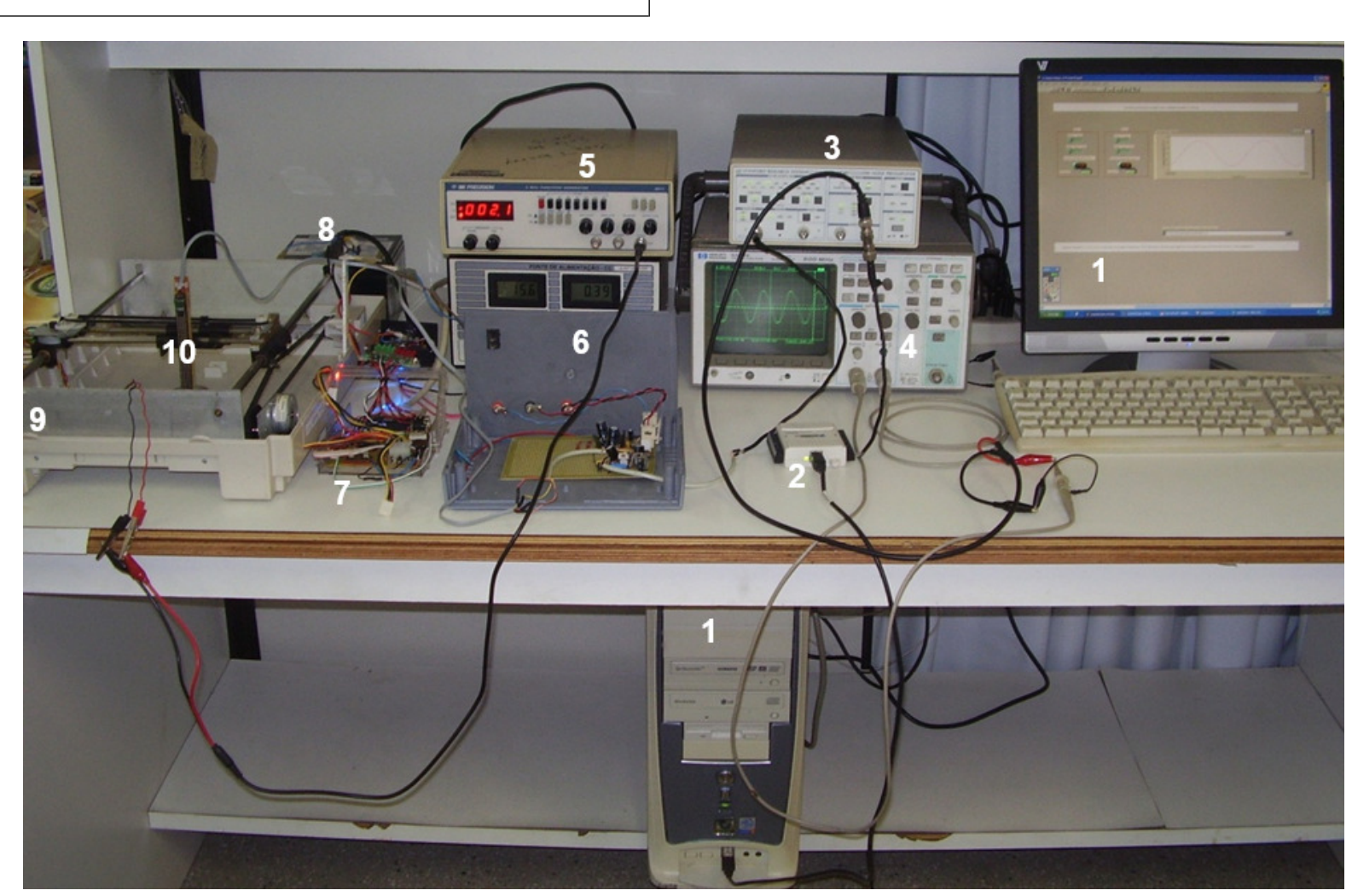

FIGURA 5: Foto do protótipo de Scanner Magnético para a obtenção de imagens magnética bidimensionais. 
Os sinais foram digitalizados em modo diferencial, por uma placa de aquisição (NI-USB6009) (2), com uma resolução de 14 bits, conectada via serial a um computador (1). Em (4) está o osciloscópio e em (5), 0 gerador de funções. A sensibilidade do sensor magnetorresistivo, comprovada por estudos anteriores, foi de $100 \mathrm{mV} / \mathrm{mT}$ e a densidade de ruído, de 44 $\mathrm{pT} /(\mathrm{Hz}) 1 / 2$, à temperatura ambiente $\left(25^{\circ} \mathrm{C}\right)$.
Na Fig. 6, apresentamos a interface gráfica utilizada na visualização da forma de onda dos sinais magnéticos medidos experimentalmente. É através desta interface que introduzimos alguns parâmetros de entrada, tais como taxa de aquisição, número de amostras, ganho digital e endereçamento dos dados adquiridos, para que sejam armazenados e, posteriormente, analisados.

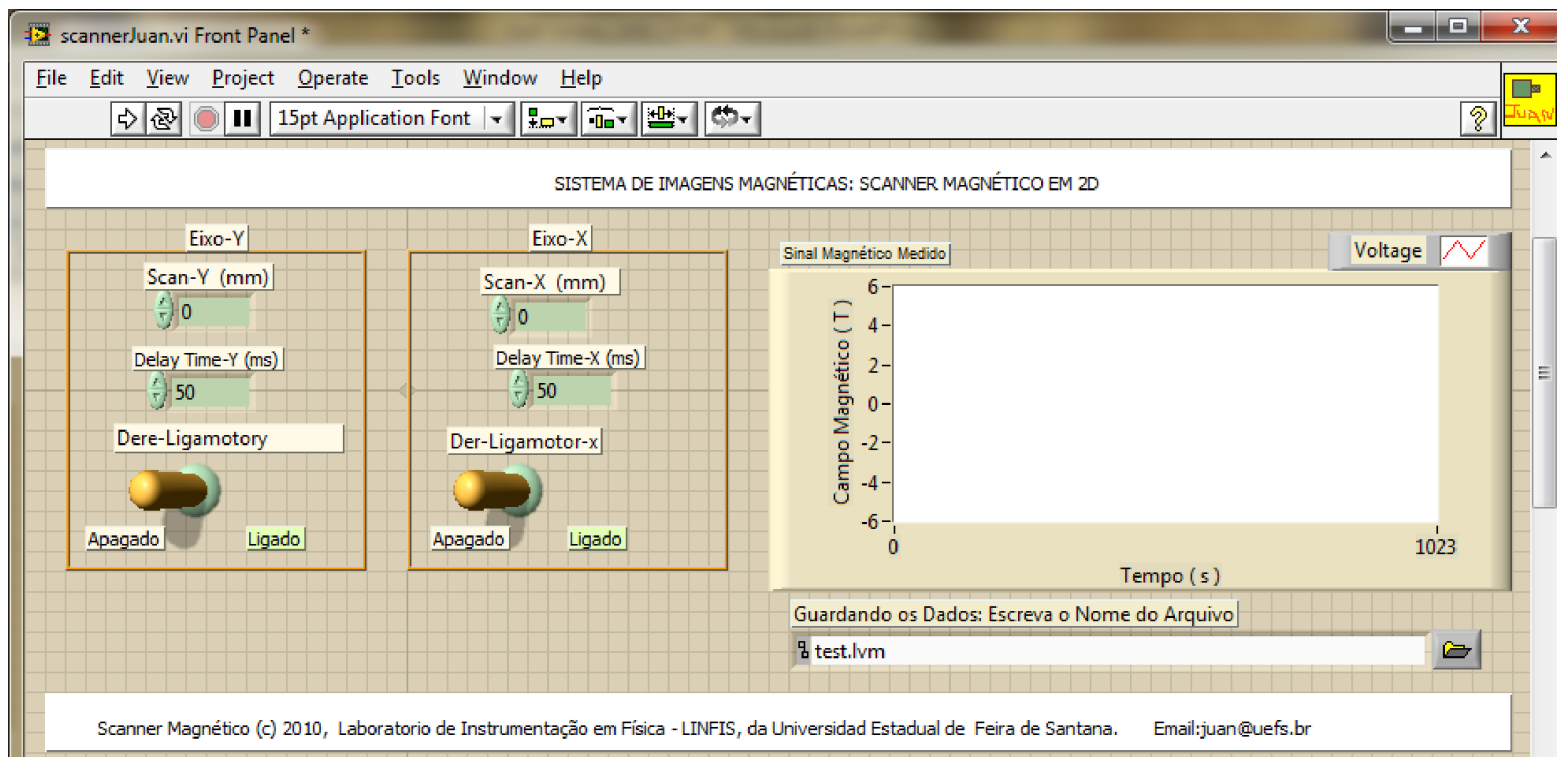

FIGURA 6: Interface gráfica em LabVIEW para o controle automático do Scanner Magnético.

\section{RESULTADOS E DISCUSSÕES}

Inicialmente, foram realizados dois experimentos; o primeiro, foi o estudo da dependência do campo magnético com a posição, no qual o detector se deslocou ao longo do eixo- $X$, passando acima das amostras magnéticas; e, o segundo, foi a obtenção de uma imagem magnética em duas dimensões de um imã cilíndrico.

\section{A. Campo Magnético versus Posição}

Na Fig. 7a, mostramos o sinal do campo magnético, medido experimentalmente pelo Scanner Magnético ao passar por um ímã de neodímio em forma de anel (ver Fig. 1a), para uma distância sensor-fonte de $46 \mathrm{~mm}$. Os valores de picos da indução do campo magnético detectado foram da ordem de $\pm 5 \mu \mathrm{T}$ e a forma de onda parece ser afetada pela geometria da amostra. Na Fig. 7b, mostramos o sinal do campo magnético, medido experimentalmente pelo Scanner Magnético ao passar por ímã de Ferrita em forma de anel (ver Fig. 1b), para uma distância sensor-fonte de $40 \mathrm{~mm}$. A forma de onda na sua primeira parte mostra os valores positivos do campo, evidenciando a presença do 
polo norte e, depois, o polo sul.

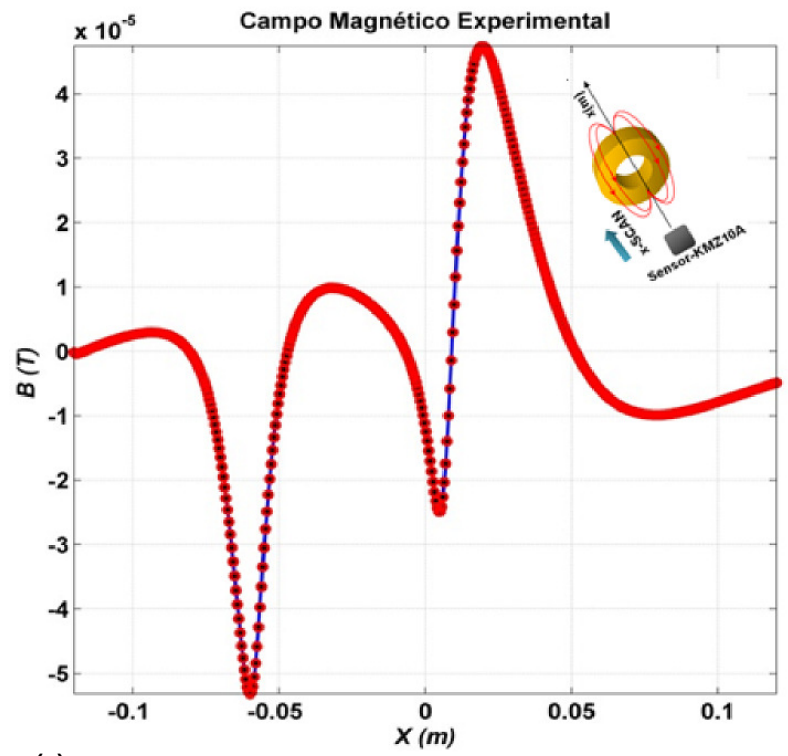

(a)

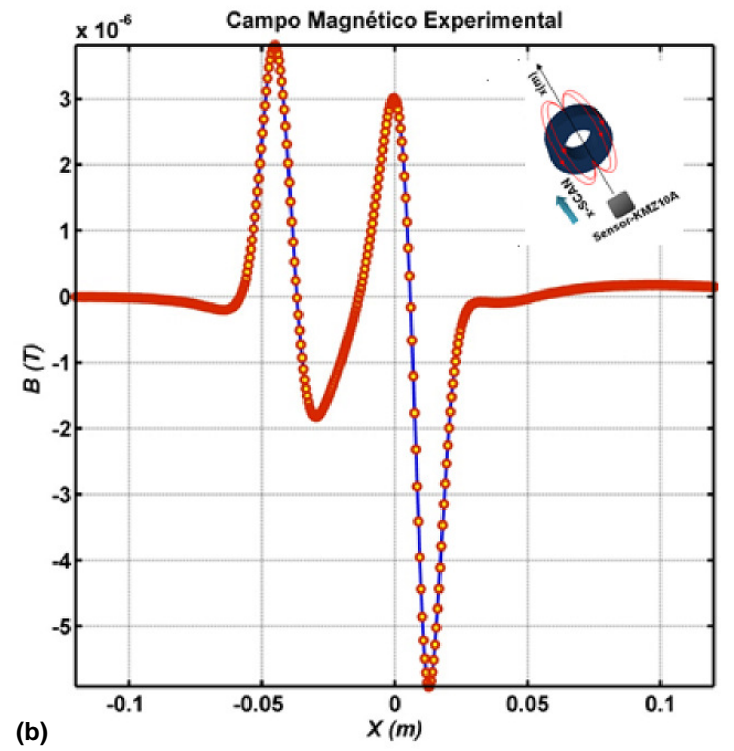

FIGURA 7: Sinais do campo magnético em função do espaço, usando imã de (a) neodímio e, (b) de ferrite em formato de anel.

Entretanto, independentemente disso, é muito semelhante à forma de onda obtida na Fig. 7a, também geradas por uma amostra com a mesma geometria. Isto mostra a capacidade do scanner de reproduzir resultados experimentais para amostras com as mesmas características espaciais. Os valores de pico positivo e negativo da indução do campo magnético detectado foram da ordem de $4 \mu \mathrm{T}$ e $-6 \mu \mathrm{T}$, respectivamente.
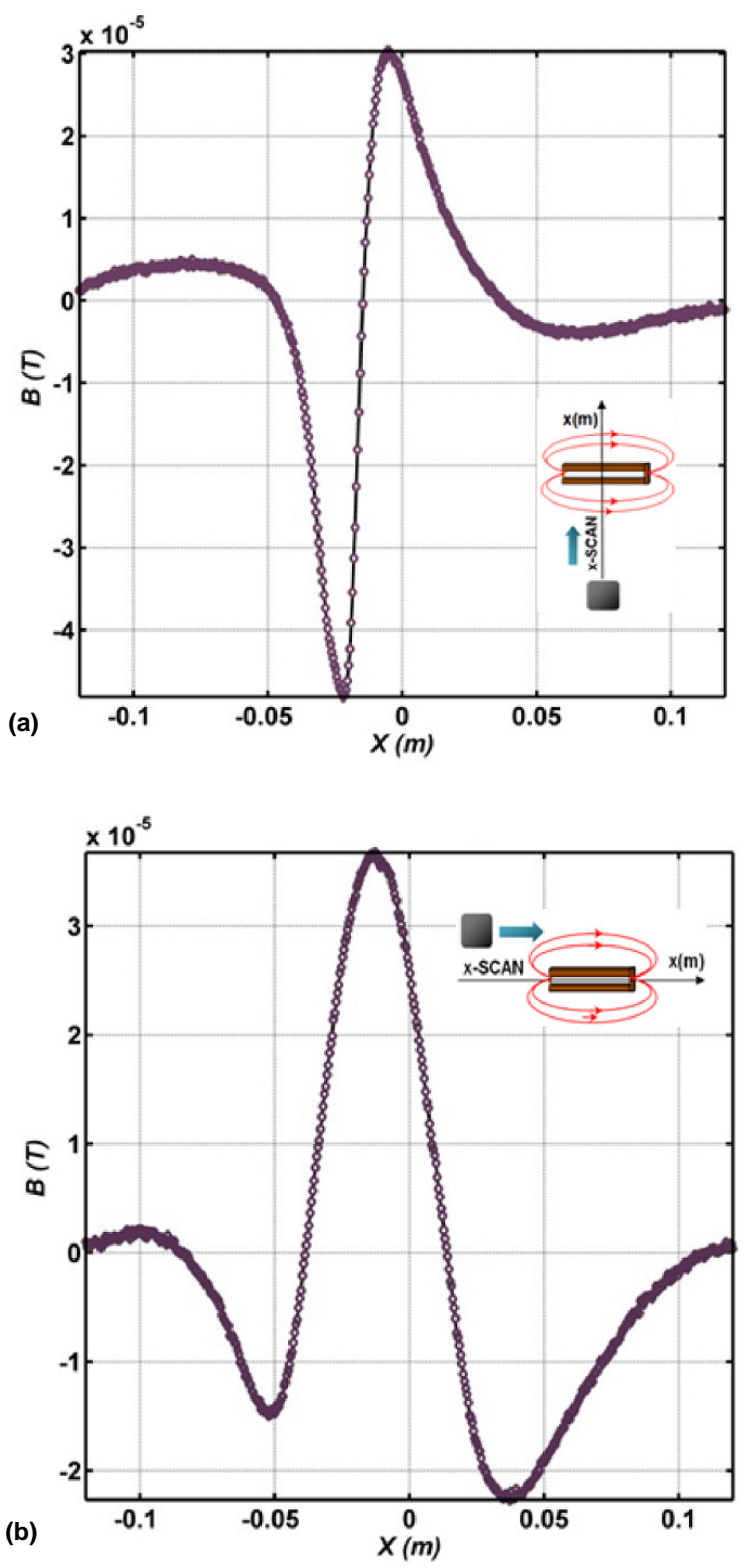

FIGURA 8: Campo magnético medido em função da posição, gerado por um imã cilíndrico de dimensões $(4 \times 2) \mathrm{mm}$, a uma distância do sensor fonte de $46 \mathrm{~mm}$. 
Na Fig. (8) são apresentados os sinais do campo magnético gerado por um ímã de ferrite com geometria de bloco retangular de dupla camada (ver Fig. 1e) em função da distância. Na Fig. 8a, mostramos o mesmo sinal, porém, agora, para uma distância Liftoff de $5 \mathrm{~mm}$. Desse gráfico, vê-se que as amplitudes dos sinais medidos são da ordem de 3 $\mu \mathrm{T}$, para o pico positivo (polo sul), e de -4.5 $\mu \mathrm{T}$, para o pico negativo (polo norte). Na Fig. 8b, o bloco é posicionado na direção horizontal à direção do eixo- $X$, para uma separação de Liftoff de $10 \mathrm{~mm}$. Neste último caso, a forma de onda é diferente (dipolar) e o valor do campo magnético é da ordem de $4 \mu \mathrm{T}$ e $-2.4 \mu \mathrm{T}$, para os maiores picos (positivo e negativo) do campo magnético medido, respectivamente.

Na Fig. (9) são apresentados os sinais do campo magnético gerado por um ímã de Ferrita com um orifício de $5 \mathrm{~mm}$ na parte central e medidos para duas distâncias Litoffs diferentes. Na Fig. 9a, as medições foram realizadas para uma distância sensor-fonte de $15 \mathrm{~mm}$, com varredura paralela ao plano do scanner (eixo$X)$. No primeiro caso, o valor máximo da indução do campo magnético foi de $3 \mathrm{mT}$ e o mínimo foi, aproximadamente, de $-4 \mathrm{mT}$. $\mathrm{Na}$ Fig. 4.7b, as medidas foram feitas para uma separação de Liftoff de $10 \mathrm{~mm}$, com o bloco fantoma girado $180^{\circ}$, observando a simetria dos polos norte e sul. Como a distância sensor-fonte foi menor, os valores de picos aumentaram para $6,5 \mathrm{mT}$ e $-6,3 \mathrm{mT}$, para os valores máximo e mínimo, respectivamente.

Foram realizadas medidas do campo magnético em função da posição. Na Fig. 10a-c, ilustramos os componentes do campo magnético, medidos enquanto o sensor se mover sobre o polo norte (Fig.10a), sul (Fig. $10 \mathrm{~b})$ e na horizontal sul-norte (Fig. 10c).

\section{B. Imagens Magnéticas Bidimensionais}

Para demonstrar a capacidade do sistema em obter imagens magnéticas, foi realizado um experimento para a registro da imagem magnética gerada por um imã cilíndrico de di- mensões $(4 \times 2) \mathrm{mm}$, localizado a uma distância sensor-fonte de $46 \mathrm{~mm}$.
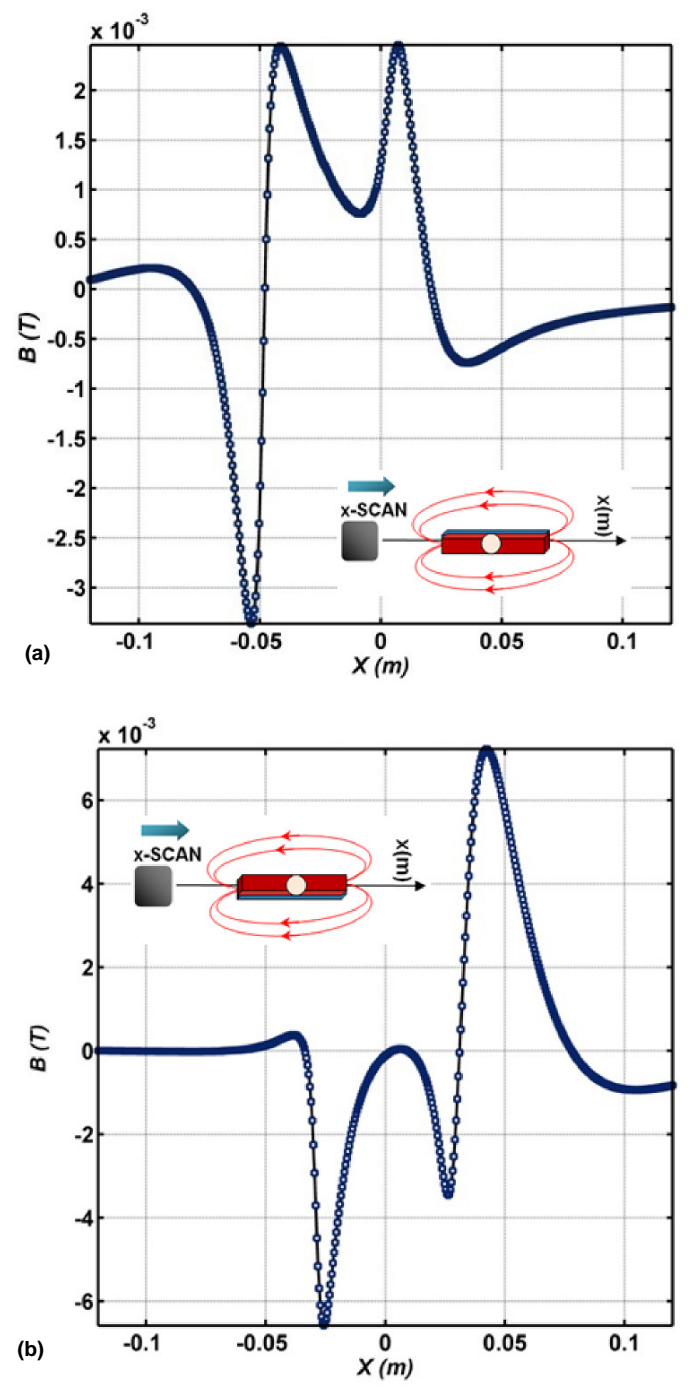

FIGURA 9: Campo magnético em função da distância, gerado por um imã de ferrite, em forma de bloco, contendo um orifício de $5 \mathrm{~mm}$ na parte central. Os campos forma medidos em duas distâncias Liftoff diferentes: (a) $15 \mathrm{~mm}$ e (b) $10 \mathrm{~mm}$, com varredura realizada paralelamente à direção do scan (eixo- $X$ ). No segundo experimento, o bloco foi girado de $180^{\circ}$.

Na Fig. 11, podemos observar todos os sinais do campo magnético medidos experimentalmente pelo Scanner Magnético, em função da distância ao longo do eixo-X. Vê-se que 
os sinais apresentaram uma excelente relação sinal/ruído. No total, foram 90 sinais ao longo do eixo-Y. Pode ser observado que o valor máximo do campo magnético foi de $40 \mu \mathrm{T}$, e $2,2 \mu \mathrm{T}$ foi o valor mínimo. Estes sinais foram agrupados num formato matricial, resultando numa matriz de $(650 \times 90)$ pixels ou pontos (dígitos), que, posteriormente, foram indexadas às distâncias de cada direção de scan X-Y e ao período espacial de amostragem (dx, dy).

A Fig. 11a, mostra uma imagem magnética bidimensional indexada a um mapa de cores falsas, também é chamada de imagem das linhas de isocampo ou contornos do campo magnético, gerada pelo dipolo magnético do fantoma (ímã cilíndrico) para uma distância sensor-fonte de $46 \mathrm{~mm}$. Na Fig. 11b, podemos ver uma vista em 3D da imagem bidimensional, representada por uma superfície de malha de pontos. O pico de campo magnético medido foi de $40 \mu \mathrm{T}$. As imagens foram obtidas off-line por meio de um script ou rotina computacional escrita na linguagem de programação MATLAB.

\section{CONCLUSÕES}

Um protótipo experimental de um Scanner Magnético foi desenhado, construído e testado preliminarmente. O Sistema de imagens tem capacidade de escanear áreas de dimensões de $(0.12 \times 0.12) \mathrm{m}^{2}$. O sistema é capaz de detectar ou produzir imagens de campos magnéticos da ordem de $10^{-6} \mathrm{~T}$ ou menores. Os resultados preliminares são apresentados e mostraram que o sistema é capaz de obter sinais e imagens magnéticas gerados por diferentes fontes magnéticas ou fantomas magnéticos, com geometrias e características diferentes. Concluímos que o Sistema Magnético adquire sinais e imagens de baixa amplitude do campo magnético e apresenta potencial para ser aplicado em estudos na área do Bioeletromagnetismo.

\section{Agradecimentos}

Este trabalho foi financiado em parte pela Universidade de Feira de Santana.
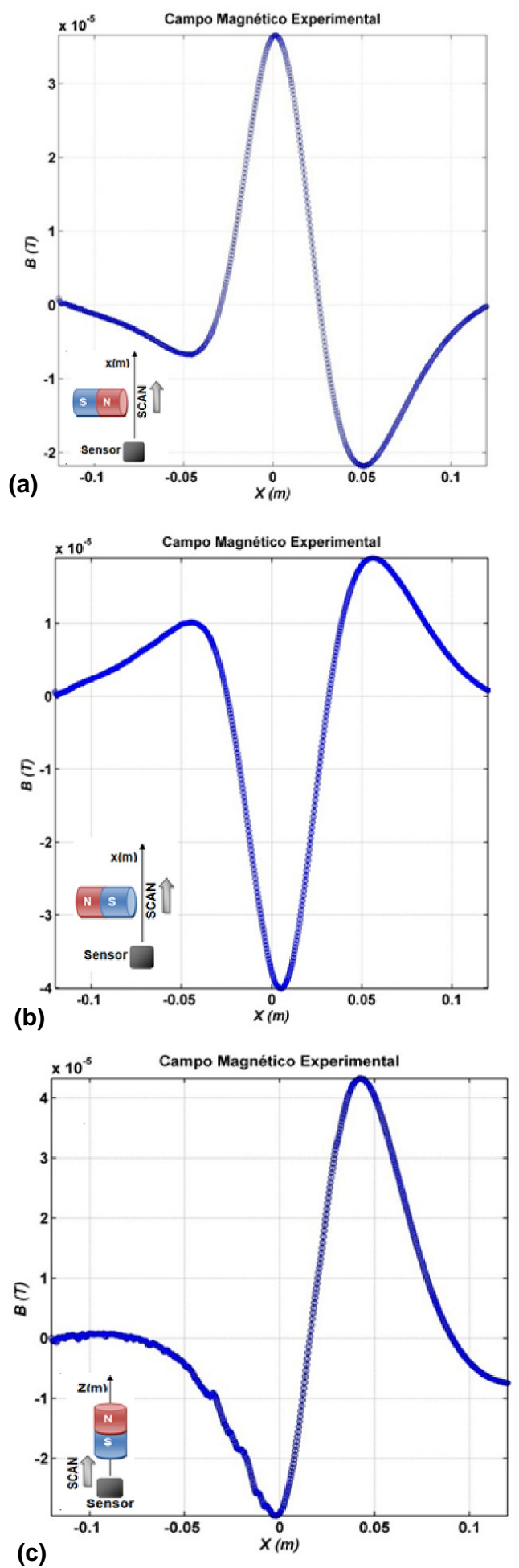

FIGURA 10: Campo magnético medido em função da posição, gerado por um imã cilíndrico de dimensões $(4 \times 2) \mathrm{mm}$, a uma distância do sensor fonte de $46 \mathrm{~mm}$. 


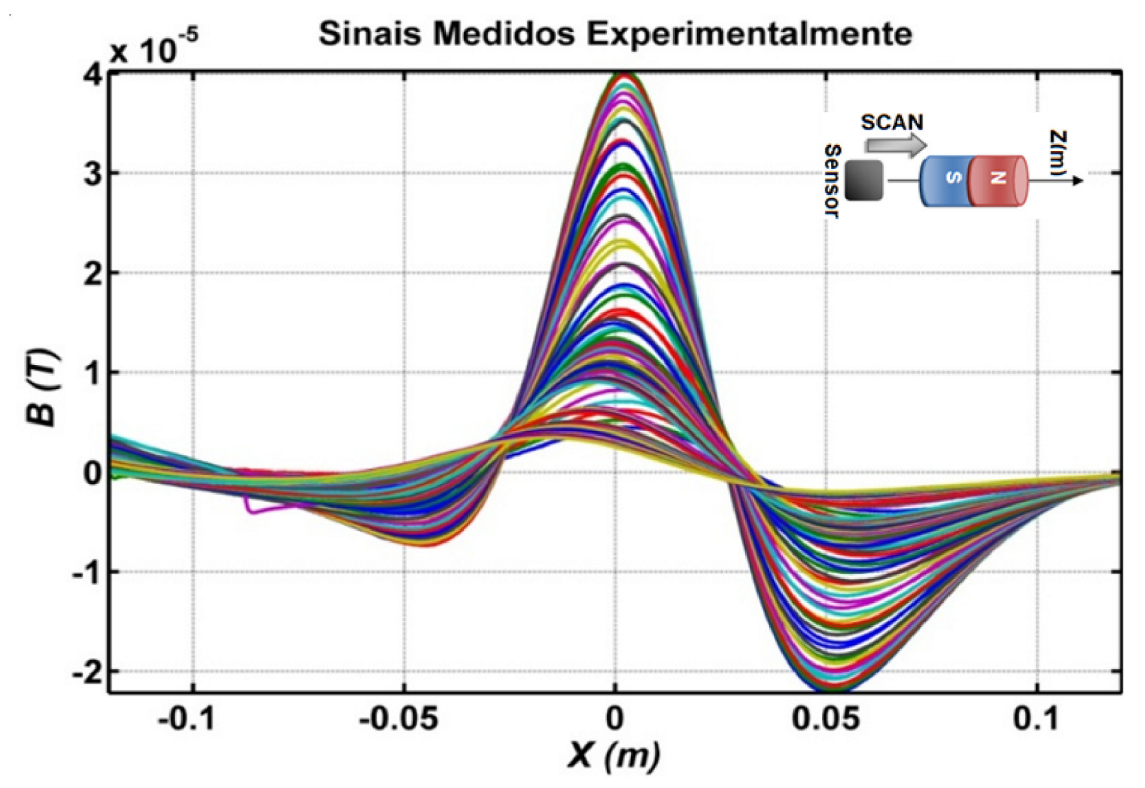

FIGURA 11: Sinais do campo magnético em função so espaço, usando-se um imã de neodímio de dimensões $(4 \times 2) \mathrm{mm}$, a uma distância do sensor fonte de $46 \mathrm{~mm}$.

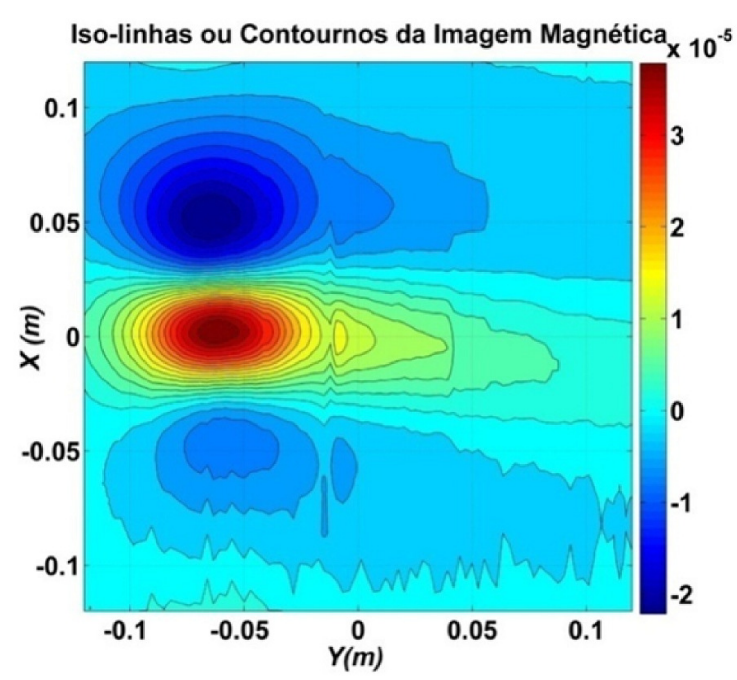

(a)

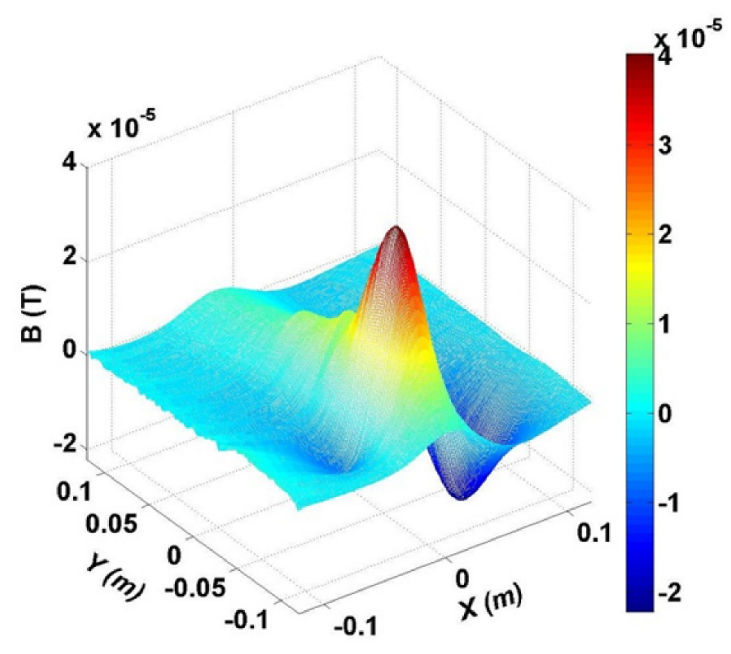

(b)

FIGURA 12: (a) Imagem bidimensional magnética ou mapa de contornos do campo magnético, e (b) Imagem magnética numa suérfície de malha de pontos (visão 3D). 
[1] C-C. Cheng, C-C. Chien, H-H. Chen, Y. Hwu, Y-T. Ching, Image Alignment for Tomography Reconstruction from Synchrotron X-Ray Microscopic Images. PLoS ONE 9, (1) e84675 (2014). http://doi:10.1371/journal.pone.0084675.

[2] Hui $\mathrm{Hu}$, Multi-slice helical CT: scan and reconstruction. Med. Phys. 26, (1) 5 (1999).

[3] H. Kado, M. Higuchi, M. Shimogawara, Y. Haruta, Y. Adachi, J. Kawai, H. Ogata, G. Uehara, Magnetoencephalogram systems developed at KIT. IEEE Trans. Appl. Supercond. 9, 4057 (1999).

[4] J. Malmivuo, R. Plonsey, Bioelectromagnetism - Principles and Applications of Bioelectric and Biomagnetic Fields. New York: BemBook-Oxford University Press (1995).

[5] D.B. de Araújo, A.A. Carneiro, E.R. Moraes, O. Baffa, Biomagnetismo uma nova interface entre a física e a biologia. Ciência Hoje 26, (153) 24 (1999).

[6] A. Gramfort et all, MNE software for processing $M E G$ and EEG data. NeuroImage 86, 446 (2014).

[7] A.A Mazzola, Ressonância magnética: princípios de formação da imagem $e$ aplicações em imagem funcional. Rev. Bras. Fis. Med. 3, (1) 117 (2009).

[8] R.W. Brown et all, Magnetic Resonance Imaging: Physical Properties and Sequence Design. New Jersey: John Wiley \& Sons (2014).

[9] F. Martelli, S. Del Bianco, A. Ismaelli, G. Zaccanti, Light propagation through biological tissue and other diffusive media: theory, solutions, and software. Bellingham: SPIE (2009).

[10] T.Z. Pavan, L.P. Neves, A.A.O. Carneiro, Elastografia por ultrassom: uma nova modalidade de imagem. Ciência Hoje 42, (251) 28 (2008).

[11] G.B. Saha, Basics of PET Imaging. Physics, Chemistry, and Regulations (2nd Edition). New York: Springer (2010).

[12] F.A. MettlerJr, M.J. Guiberteau, Essentials of Nuclear Medicine Imaging (6th Edition). Philadelphia: Saunders Elsevier (2012).
[13] M. Soleimani, W.R.B. Lionheart, Absolute Conductivity Reconstruction in Magnetic Induction Tomography Using a Nonlinear Method. IEEE Trans. Med. Imag. 25, (12) 1521 (2006).

[14] W. Hsin-Yu, M. Soleimani, A Magnetic Induction Tomography System for Prospective Industrial. Processing Applications. Chin. J. Chem. Eng. 20, (2) 406 (2012).

[15] H. Scharfeter et all, Magnetic induction tomography: single-step solution of $3 D$ inverse problem for differential image reconstruction. Int. J. Inf. Sys. Sci. 2, (4) 585 (2006).

[16] I. Kolesnikov, Magnetic Tomography Method (MTM). A Remote Non-destructive Inspection Technology for Buried and Sub Sea Pipelines. Offshore Tech. Conf. (2014). http://doi:10.4043/24569-MS.

[17] J.A. Leyva-Cruz, E.S. Ferreira, M.S.R. Miltão, A.V. Andrade-Neto, A.S. Alves, M.E. Cano, Reconstruction of magnetic source images using the Wiener filter and a multichannel magnetic imaging system. Rev. Sci. Inst. 854, 167 (2014). http://dx.doi.org/10.1063/1.4884641.

[18] P.D. Dimitropoulos, J.N. Avaritsiotis, A 2-D ferrous object imaging technique based on magnetic field sensor arrays. Sensors and Actuators A 106, (1) 336 (2003).

[19] D.J. Mapps, Remote magnetic sensing of people. Sensors and Actuators A 106, (1-3) 321 (2003). http://dx.doi.org/10.1016/S09244247(03)00193-6.

[20] J.A. Leyva-Cruz et all, Imaging Ferromagnetic Tracers with a Magnetoresistive Sensors Array. AIP Conf. Proc. 854, 167 (2006). http://dx.doi.org/10.1063/1.2356436.

[21] M.E. Cano et all, Superficial magnetic imaging by an xy-scanner of three magnetoresistive channels. Rev. Sci. Inst. 83, 033705 (2012). http://dx.doi.org/10.1063/1.3694002.

[22] S.L. Vieira, L.N. de Oliveira, A.A.O. Carneiro, Princípios físicos da elastografia por ressonância magnética. Rev. Bras. Ens. Fis 36, (2) 2301 (2014). 